\title{
RECURSIVE CALCULATION OF SURVIVAL PROBABILITIES
}

\author{
By David C. M. Dickson and Howard R. Waters \\ Heriot-Watt University, Edinburgh, United Kingdom
}

\begin{abstract}
In this paper we present an algorithm for the approximate calculation of finite time survival probabilities for the classical risk model. We also show how this algorithm can be applied to the calculation of infinite time survival probabilities. Numerical examples are given and the stability of the algorithms is discussed.
\end{abstract}

\section{KEYWORDS}

Survival probability; finite time; infinite time; recursive calculations; numerical stability.

\section{INTRODUCTION}

The primary aim of this paper is the approximate calculation of the probablity of survival in continuous and finite time for a general classical risk process. We assume, without loss of generality, that the expected number of claims per unit time for this process is 1 and that the expected amount of a single claim is also 1. This process can be characterized as follows:

- the number of claims occurring up to time $t$, denoted ${ }_{0} N_{t}$, has a Poisson distribution with parameter $t$,

- the amount of the $i$-th claim is ${ }_{0} Y_{i}$, where $\left\{{ }_{0} Y_{i}\right\}_{i=1}^{\infty}$ is a sequence of i.i.d. non-negative random variables which are also independent of the claim number process, and whose first two moments exist,

- the premium income per unit time is $1+\theta$, where $\theta$ is the premium loading factor. (We shall assume $\theta \geq 0$, but some of our later results require only that $(1+\theta)>0$.)

(We use the subscript " 0 " where appropriate to indicate that we are dealing with our initial process.) For a given initial reserve $u(\geq 0)$ we denote by ${ }_{0} \delta(u, t)$ the probability of survival in continuous time up to time $t$, so that

$$
{ }_{0} \delta(u, t)=\operatorname{Pr}\left[u+(1+\theta) \tau-\sum_{i=1}^{{ }_{0} N_{\tau}}{ }_{0} Y_{i} \geq 0 \text { for all } \tau, 0<\tau \leq t\right]
$$


Our approach to the calculation of ${ }_{0} \delta(u, t)$ is to show that ${ }_{0} \delta(u, t)$ can be approximated by the probability of survival in discrete and finite time for a particular risk process, and then to discuss the calculation of this latter probability. The particular risk process we use is a classical risk process characterized as follows:

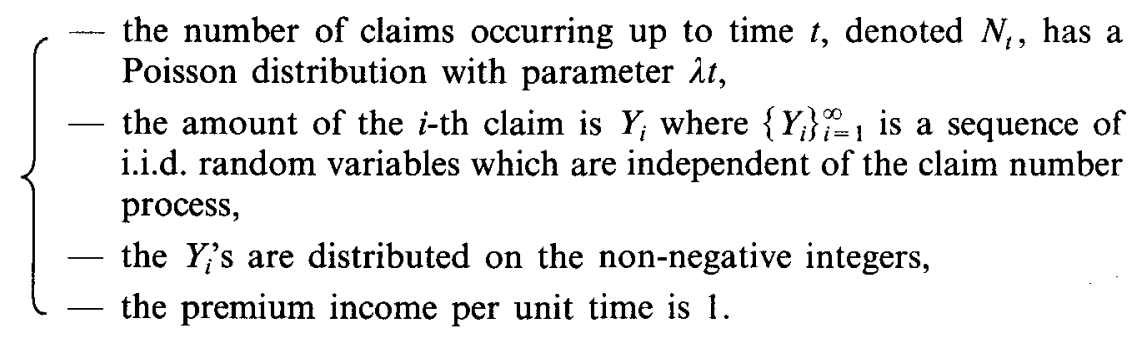

We introduce the following notation for this particular risk process:

$$
\begin{aligned}
& b_{k}=\operatorname{Pr}\left[Y_{i}=k\right] \quad \text { for } \quad k=0,1,2, \ldots \\
& \delta(u, t)=\operatorname{Pr}\left[u+\tau-\sum_{i=1}^{N_{\tau}} Y_{i} \geq 0 \quad \text { for } \quad \tau=1,2, \ldots, t\right]
\end{aligned}
$$

so that $\delta(u, t)$ denotes the probability of survival in discrete time up to time $t$ for this particular risk process, given initial reserve $u$, which we always assume to be non-negative. With suitable choices for $\lambda$ and the $b_{k}$ 's we can then argue that

$$
{ }_{0} \delta(u, t) \simeq \delta(u \beta,(1+\theta) \beta t)
$$

for some positive constant $\beta$.

Formula (1.3) can be justified by using a discretizing and re-scaling argument as follows:

STEP 1 Discretize the initial process:

Let $\left\{{ }_{1} Y_{i}\right\}_{i=1}^{\infty}$ be a sequence of i.i.d. random variables distributed on the discrete points $0,1 / \beta, 2 / \beta, \ldots$, for some $\beta>0$, in such a way that the distribution of ${ }_{1} Y_{i}$ approximates to that of ${ }_{0} Y_{i}$.

Let $\quad b_{k}=\operatorname{Pr}\left[{ }_{1} Y_{i}=\mathrm{k} / \beta\right]$ for $k=0,1,2, \ldots$

Let

$$
{ }_{1} \delta(u, t)=\operatorname{Pr}\left[u+(1+\theta) \tau-\sum_{i=1}^{{ }_{0} N_{z}}{ }_{1} Y_{i} \geq 0 \text { for all } \tau, 0<\tau \leq t\right]
$$

so that ${ }_{1} \delta(u, \mathrm{t})$ is the probability of survival in continuous time before time $t$, given initial reserve $u(\geq 0)$, for the initial process but with ${ }_{0} Y_{i}$ replaced by the discrete random variable ${ }_{1} Y_{i}$. 
Then, if ${ }_{1} Y_{i}$ is a "good" approximation to ${ }_{0} Y_{i}$,

$$
{ }_{1} \delta(u, t) \simeq{ }_{0} \delta(u, t) .
$$

STEP 2 Change the monetary unit:

Define ${ }_{2} Y_{i}$ to be equal to $\beta Y_{i}$, so that

$$
\operatorname{Pr}\left[{ }_{2} Y_{i}=k\right]=b_{k} \quad \text { for } \quad k=0,1,2, \ldots
$$

Denoting by ${ }_{2} \delta(w, t)$ the probability

$$
\operatorname{Pr}\left[w+(1+\theta) \beta \tau-\sum_{i=1}^{0 N_{\tau}}{ }_{2} Y_{i} \geq 0 \text { for all } \tau, 0<\tau \leq t\right]
$$

it can be seen that

$$
{ }_{1} \delta(u, t)={ }_{2} \delta(u \beta, t)
$$

and hence

$$
{ }_{0} \delta(u, t) \simeq{ }_{2} \delta(u \beta, t)
$$

STEP 3 Change the time unit:

Let ${ }_{3} N_{t}$ be a Poisson process with parameter $\lambda=1 /[(1+\theta) \beta]$.

Let $\quad{ }_{3} \delta(w, t)=\operatorname{Pr}\left[w+\tau-\sum_{i=1}^{3 N_{\tau}}{ }_{2} Y_{i} \geq 0\right.$ for all $\left.\tau, 0<\tau \leq t\right]$.

Then it can be seen that

$$
{ }_{2} \delta(w, t)={ }_{3} \delta(w,(1+\theta) \beta t)
$$

and hence that

$$
{ }_{0} \delta(u, t) \simeq{ }_{3} \delta(u \beta,(1+\theta) \beta t) .
$$

Finally in our argument to justify (1.3), note that the risk process emerging from STEP 3 is the risk process characterized by $(1.2)$ and that $\delta(u, t)$ is the discrete time probability of survival corresponding to ${ }_{3} \delta(u, t)$. Intuitively, $\delta(u, t)$ should be a good approximation to ${ }_{3} \delta(u, t)$ if, for a given $t$, the number of re-scaled time units, $(1+\theta) \beta t$, is large, so that there are frequent checks for survival in the discrete case.

For the remainder of this paper our theoretical work will be based on the risk process characterized by (1.2). We introduce the following notation for this process :

$X_{n}$ denotes the aggregate claims from time $n-1$ to time $n$, so that

$$
X_{n}=\sum_{i=N_{n-1}+1}^{N_{n}} Y_{i} \text { for } n=1,2, \ldots\left(=0 \text { if } N_{n-1}=N_{n}\right)
$$


$g_{k}$ is the probability that $X_{n}$ takes the value $k$, for $k=0,1,2 \ldots$

$m_{k}$ is the $k$-th moment about zero of an individual claim amount

$Z_{n}$ is the accumulated surplus up to time $n$, given initial surplus $u \geq 0$, so that

$$
Z_{n}=u+n-\sum_{i=1}^{n} X_{i} \quad \text { for } n=1,2, \ldots
$$

Note that since $Y_{i}$ is distributed on the non-negative integers we can evaluate the $g_{k}$ 's using the recursive method of PANJER (1981). We shall assume for the remainder of this paper that the $g_{k}$ 's are known and that $u$ is a non-negative integer. Note also that $Z_{n+1}$ can take only the values

$$
\begin{array}{ll}
Z_{n}+1 & \text { (if } \left.X_{n+1}=0\right) \\
Z_{n} & \text { (if } \left.X_{n+1}=1\right) \\
Z_{n}-1 & \text { (if } X_{n+1}=2 \text { ) }
\end{array}
$$

\section{THE METHOD OF De Vylder AND GoOvaerTs}

DE VyLDER and GoovaerTs (1988) present a very neat recursive algorithm for the approximate calculation of ${ }_{0} \delta(u, t)$. Their method involves discretizing the risk process and then re-scaling it, in almost exactly the same way as we have described in our Section 1. In terms of the process characterized by (1.2), their algorithm is as follows:

$$
\begin{aligned}
& \delta(w, 1)=\sum_{j=0}^{w+1} g_{j} \quad \text { for } \quad w=0,1,2, \ldots \\
& \delta(w, m)=\sum_{j=0}^{w+1} \delta(w+1-j, m-1) g_{j} \quad \text { for } \quad w=0,1, \ldots \\
& \text { and } m=2,3, \ldots
\end{aligned}
$$

The rationale behind this algorithm is as follows:

- $\delta(w, 1)$ can be calculated directly from (2.1) since the $g_{k}$ 's are known,

- for $m \geq 2, \delta(w, m)$ can be calculated by conditioning on the surplus after 1 unit of time; with probability $g_{j}$ this surplus is $(w+1-j)$ and the probability of survival over a further $(m-1)$ units of time is $\delta(w+1-j, m-1)$.

In terms of the calculations involved, formula (2.2) can perhaps be most easily appreciated by considering Figure 1. We suppose that we wish to calculate $\delta(u, t)$ for some given $u$ and (positive integer) $t(>1)$. We first calculate $\delta(w, 1)$ for $w=0,1, \ldots, u+t-1$ using (2.1). We then use (2.2) to calculate $\delta(w, 2)$ for $w=0,1,2, \ldots, u+t-2$. In general, we calculate $\delta(w, \tau)$ for $w=0,1,2, \ldots, u+t-\tau$ having first calculated $\delta(w, \tau-1)$ for 


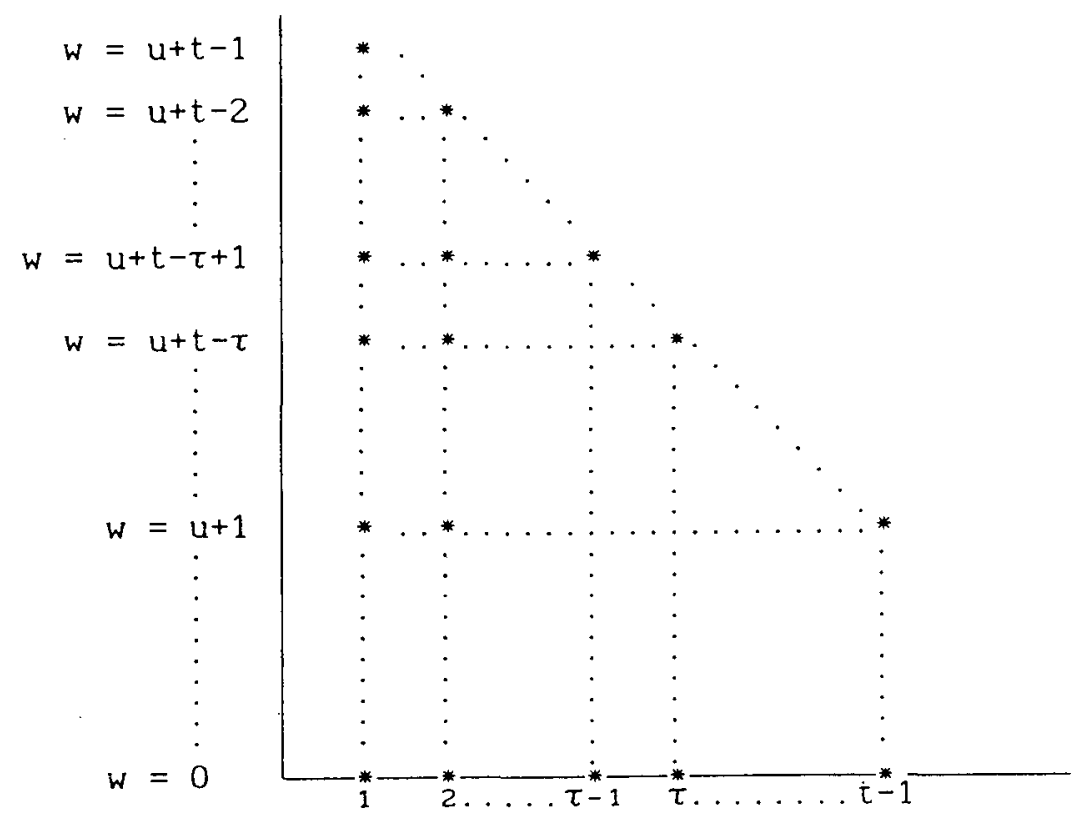

FIGURE 1. Combinations of $w$ and $\tau$ for which values of $\delta(w, \tau)$ are required to calculate $\delta(u, t)$ using the method of De Vylder and Goovaerts.

$w=0,1,2, \ldots, u+t-\tau+1$. It can be seen that to calculate $\delta(u, t)$ we have, at least in principle, to calculate $\delta(w, \tau)$ for all values of $(w, \tau)$ in the trapezoidal area given by $1 \leq \tau \leq t-1$ and $0 \leq w \leq u+t-\tau$.

There is one respect in which the above description represents a refinement of the algorithm presented by DE VYLDER and GoovaERTs (1988). In their Section 7 they state that, "We can adopt any unit of money and any unit of time." However, re-scaling of the time unit results in a premium income per unit time which can be greater than 1; our re-scaling of the time unit, as described in our Section 1, results in a premium income per unit time which is equal to 1 .

There are two respects in which the above description is a simplification of De Vylder and Goovaerts's algorithm. These are:

1. Truncation: De Vylder and Goovaerts (1988, Sections 4 and 5) point out that the algorithm as described above requires a lot of calculations to be carried out and hence requires a considerable amount of computer time. They propose, and use, a method for reducing the number of calculations required in such a way that the error resulting from this approximation can be bounded.

2. Averaging: De Vylder and Goovaer Ts (1988, formula (1)) point out that, in the notation of our Section 1,

$$
\delta(u-1, t) \leq{ }_{3} \delta(u, t) \leq \delta(u, t)
$$


and, in their numerical example, they propose approximating ${ }_{3} \delta(u, t)$ not by $\delta(u, t)$ but by $\bar{\delta}(u, t)$ where

$$
\bar{\delta}(u, t)=\frac{1}{2}\{\delta(u-1, t)+\delta(u, t)\}
$$

with $\delta(u-1, t)$ taken to be zero if $u$ is zero.

A numerical example: Table 1 shows values of ${ }_{0} \delta(u, t)$ for various combinations of $u$ and $t$ for the risk process with exponentially distributed individual claims and with two values for the premium loading factor $\theta$, viz. 0.1 and 0.2 . The key to Table 1 is as follows:

(1) denotes the exact value of ${ }_{0} \delta(u, t)$ as given by WIKSTAD (1971);

(2) denotes the approximation to ${ }_{0} \delta(u, t)$ given by DE VYLDER and GooVAERTS (1988, Table 1);

(3) denotes the approximation to ${ }_{0} \delta(u, t)$ given by (2.1) and (2.2) above.

TABLE 1 (See Section 2 for details)

(a) Premium loading factor $\theta=0.1$

\begin{tabular}{lllll}
\hline \hline & & $t=1$ & $t=10$ & $t=100$ \\
\hline$u=0$ & $(1)$ & 0.5366 & 0.2146 & 0.1100 \\
& $(2)$ & 0.3401 & 0.1562 & 0.0814 \\
& $(3)$ & 0.5515 & 0.2239 & 0.1150 \\
$u=1$ & $(1)$ & 0.7619 & 0.3874 & 0.2052 \\
& $(2)$ & 0.4159 & 0.2322 & 0.1252 \\
& $(3)$ & 0.7699 & 0.3953 & 0.2098 \\
$u=10$ & $(1)$ & 0.9997 & 0.9681 & 0.7395 \\
& $(2)$ & 0.9996 & 0.9663 & 0.7366 \\
& $(3)$ & 0.9997 & 0.9687 & 0.7413 \\
\hline
\end{tabular}

(b) Premium loading factor $\theta=0.2$

\begin{tabular}{lllll}
\hline \hline & & $t=1$ & $t=10$ & $t=100$ \\
\hline$u=0$ & $(1)$ & 0.5490 & 0.2523 & 0.1717 \\
& $(2)$ & 0.3498 & 0.1829 & 0.1262 \\
& $(3)$ & 0.5636 & 0.2624 & 0.1789 \\
$u=1$ & $(1)$ & 0.7695 & 0.4356 & 0.3040 \\
& $(2)$ & 0.4212 & 0.2602 & 0.1847 \\
& $(3)$ & 0.7772 & 0.4437 & 0.3094 \\
$u=10$ & $(1)$ & 0.9997 & 0.9759 & 0.8601 \\
& $(2)$ & 0.9996 & 0.9743 & 0.8573 \\
& $(3)$ & 0.9997 & 0.9764 & 0.8615 \\
\hline
\end{tabular}


The following points should be noted concerning Table 1:

(i) The same discretization has been used to calculate (2) and (3). This is the discretization given by DE VYLDER and GoOvAERTS (1988, Section 8); in particular the parameter $\beta$ has been taken to be 20 .

(ii) Both (2) and (3) have been calculated using the truncation proposed by $\mathrm{DE}$ Vylder and GoovaerTs (1988, Sections 4 and 5) with the same truncation parameter in each case.

(iii) The figures shown for (2) have been calculated using formula (2.3), i.e. by "averaging". The figures for (3) have not been calculated using (2.3). If (2.3) had been used to calculate the figures for (3) the effects would have been an improvement in the approximation to ${ }_{0} \delta(u, t)$ for $u>0$ (e.g. the approximation to $0 \delta(10,10)$ with $\theta=0.1$ would have been 0.9684$)$ but a much worse approximation to ${ }_{0} \delta(0, t)$ (e.g. for $\theta=0.2$ the approximation to ${ }_{0} \delta(0,10)$ would have been 0.1312$)$.

(iv) The important difference in the calculation of the values for (2) and (3) is the difference in the re-scaling of the time unit, as explained above.

(v) For all combinations of $\theta, u$ and $t$ shown in Table 1, the approximation given by (3) is much closer to the exact value than is the approximatoin given by (2). We consider this to be a consequence of point (iv) above.

\section{A NEW APPROACH TO THE CALCULATION OF $\delta(u, t)$}

In this section we present an approach to the calculation of $\delta(u, t)$ different to that of Section 2. The starting point for this approach is formula (2.2). For

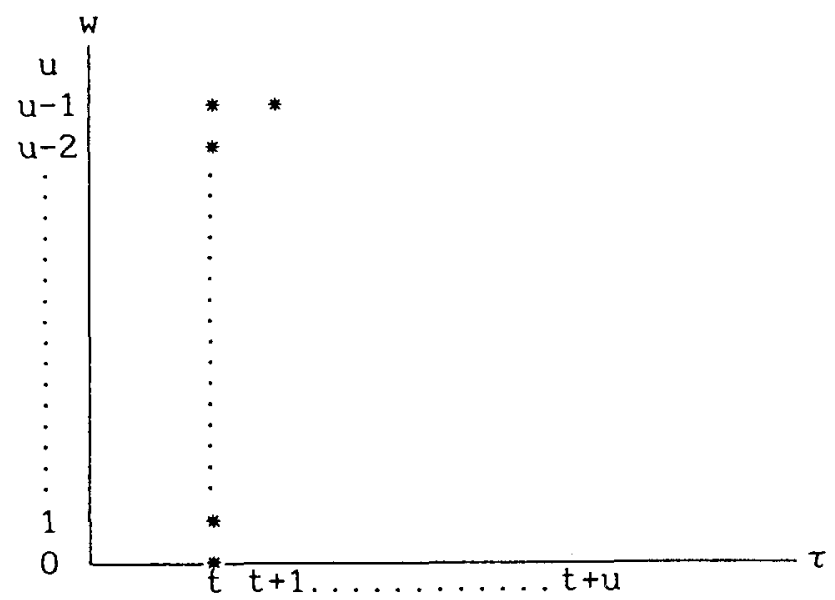

Figure 2. Combinations of $w$ and $\tau$ for which values of $\delta(w, \tau)$ are required to apply formula (3.2). 
$u>1$ and $t>0$, we can rewrite (2.2) as

$$
\delta(u-1, t+1)=\sum_{i=0}^{u} g_{i} \delta(u-i, t)
$$

To apply this approach, we do not need a formula corresponding to (2.1), but we have to consider the situation when $u=1$. This is considered in detail in Section 4. On rearranging (3.1), we see that

$$
\delta(u, t)=g_{0}^{-1}\left[\delta(u-1, t+1)-\sum_{i=1}^{u} g_{i} \delta(u-i, t)\right]
$$

Figure 2 illustrates the survival probabilities required in order to calculate $\delta(u, t)$ from (3.2). By repeated application of this approach, we see that all values of $\delta(w, \tau)$ for $w=0,1, \ldots, u-1$ and $\tau=t, t+1, \ldots, t+u-w$ are required to calculate $\delta(u, t)$. Note that all values of $\delta(0, \tau)$, $\tau=t, t+1, \ldots, t+u$, are required, but these cannot be calculated from (3.2). These values are central to our algorithm and, for the moment, we assume that these values are known. A method for finding these values is considered in Section 4.

Figure 3 illustrates the combinations of $w$ and $\tau$ for which values of $\delta(w, \tau)$ are required in order to calculate $\delta(u, t)$. The algorithm starts by calculating $\delta(1, \mathrm{t}+\mathrm{u}-1)$ from $\delta(0, t+u)$ and $\delta(0, t+u-1)$. Survival probabilities at time $t+u-2$ are then calculated, firstly $\delta(1, t+u-2)$, then $\delta(2, t+u-2)$. We next calculate survival probabilities at time $t+u-3$ and continue in this manner until we finally calculate survival probabilities at time $t$.

Calculation of $\delta(u, t)$ by this method requires that a total of $0.5 u(u+3)$ survival probabilities must first be calculated. What is remarkable about this number is that it is independent of $t$. This contrasts with the algorithm discussed in Section 2, where the number of $\delta$ values required to calculate

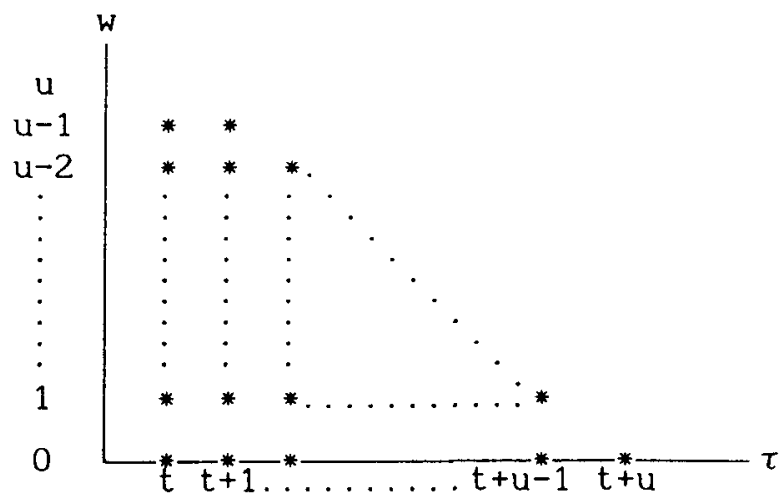

FIGURE 3. Combinations of $w$ and $\tau$ for which values of $\delta(w, \tau)$ are required to calculate $\delta(u, t)$ by repeated application of formula (3.2). 
$\delta(u, t)$ is $(t-1)(u+0.5 t+1)$, which clearly depends on $t$. However, as we shall show in Section 4, the number of calculations required to produce a value for $\delta(0, t)$ does depend on $t$.

A further difference between this algorithm and that of Section 2 is that, using the approach of De Vylder and Goovaerts, the survival probabilities required to calculate $\delta(u, t)$ are all for time periods less than $t$. The new algorithm uses survival probabilities for time periods of at least $t$. This difference is not important if we are only interested in calculating the survival probability for one particular combination of $u$ and $t$. De Vylder and Goovaerts' approach to calculting $\delta(u, t)$ also produces values of $\delta(u, j)$, for $j=1,2, \ldots, t-1$. Our new algorithm produces values of $\delta(j, t)$, for $j=0,1, \ldots, u-1$ (and the method of De Vylder and Goovaerts produces all the figures required to calculate these survival probabilities).

\section{A FORMULA FOR $\delta(0, t)$}

Let us first consider a survival probability that is slightly different to $\delta(u, t)$. Define

$$
\delta^{*}(u, t)=\operatorname{Pr}\left[u+\tau-\sum_{i=1}^{N_{\tau}} Y_{i} \geq 1 \quad \text { for } \quad \tau=1,2, \ldots, t\right]
$$

so that survival occurs only if the reserve level is strictly positive at each duration from 1 through to $t$, but the initial reserve level could be zero. When $t$ is infinite, we shall write $\delta^{*}(u)$ rather than $\delta^{*}(u, \infty)$.

Let us consider $\delta^{*}(0, t+1)$, where $t>0$. Since the initial reserve level is zero, survival under the definition of $\delta^{*}$ can occur only if there are no claims in the first unit of time. Hence

$$
\begin{aligned}
\delta^{*}(0, t+1) & =g_{0} \delta^{*}(1, t) \\
& =g_{0} \operatorname{Pr}\left[1+\tau-\sum_{i=1}^{N_{\tau}} Y_{i} \geq 1 \quad \text { for } \quad \tau=1,2, \ldots, t\right] \\
& =g_{0} \operatorname{Pr}\left[\tau-\sum_{i=1}^{N_{\tau}} Y_{i} \geq 0 \quad \text { for } \quad \tau=1,2, \ldots, t\right] \\
& =g_{0} \delta(0, t)
\end{aligned}
$$

We can use results given in GERBER (1980, pp. 19-22) for stochastic processes with exchangeable increments to find a formula for $\delta^{*}(0, t)$, and hence $\delta(0, t)$. We have that

$$
\delta^{*}(0, t)=\sum_{j=1}^{t} \operatorname{Pr}\left[Z_{n}>0, \quad \text { for } \quad n=1,2, \ldots, t-1 \quad \text { and } \quad Z_{t}=j\right]
$$


where $Z_{n}$ is as in Section 1 (with $u=0$ ). Using the duality principle,

$$
\operatorname{Pr}\left[Z_{n}>0, \text { for } \begin{array}{rl}
n= & \left.1,2, \ldots, t-1 \quad \text { and } \quad Z_{t}=j\right] \\
= & \operatorname{Pr}\left[Z_{n}<Z_{t} \text { for } n=1,2, \ldots, t-1 \quad\right. \text { and } \\
& \left.Z_{t}=j\right]
\end{array}\right.
$$

and since the process $\left\{Z_{n}\right\}$ is skipfree upwards, we have that

$\operatorname{Pr}\left[Z_{n}<Z_{t} \quad\right.$ for $\quad n=1,2, \ldots, t-1 \quad$ and $\left.\quad Z_{t}=j\right]=\frac{j}{t} \operatorname{Pr}\left[Z_{t}=j\right]$

Thus, $\delta^{*}(0, t)=\sum_{j=1}^{t} \frac{j}{t} \operatorname{Pr}\left[Z_{t}=j\right]$ and $\delta(0, t)=g_{0}^{-1} \sum_{j=1}^{t+1} \frac{j}{t+1} \operatorname{Pr}\left[Z_{t+1}=j\right]$.

Define $S_{t}$ to be aggregate claims up to time $t$, so that $S_{t}=\sum_{n=1}^{t} t y_{n}$. Let $F(j$,

$=\operatorname{Pr}\left[S_{t} \leq j\right]$ and let $f(j, t)=\operatorname{Pr}\left[S_{t}=j\right]$, for $j=0,1,2, \ldots$ Since the initial surplus is zero, $Z_{t+1}=j \Rightarrow S_{t+1}=t+1-j$, so that

$$
\begin{aligned}
\delta(0, t) & =g_{0}^{-1} \sum_{j=1}^{t+1} \frac{j}{t+1} f(t+1-j, t+1) \\
& =g_{0}^{-1} \sum_{j=0}^{t} \frac{1}{t+1} F(j, t+1)
\end{aligned}
$$

Note that since $S_{t}$ has a compound Poisson distribution with individual claims distributed on the non-negative integers, $F(j, t)$ can be calculated using PANJER'S (1981) recursion formula.

It is interesting to note that the formula for $\delta^{*}(0, t)$ can also be expressed in terms of $F(j, t)$ as

$$
\delta^{*}(0, t)=\frac{1}{t} \sum_{j=0}^{t-1} F(j, t)
$$

This expression is clearly analogous to the well known formula for ${ }_{0} \delta(0, t)$ for the general risk process specified by (1.1), as given in, e.g., SEAL (1978, p. 48).

\section{SOME NUMERICAL EXAMPLES AND SOME COMMENTS ON NUMERICAL STABILITY}

\subsection{Numerical examples using the algorithm in Sections 3 and 4}

Table 2 shows values of, and approximations to, ${ }_{0} \delta(u, t)$ for a risk process with exponentially distributed individual claims and premium loading factor, $\theta$, equal to 0.1 . The key to Table 2 is as follows:

(1) denotes the exact value of ${ }_{0} \delta(u, t)$, as given by SEAL (1978, Table 2.4), 
(2) denotes the approximation to ${ }_{0} \delta(u, t)$ calculated using the algorithm discussed in Sections 3 and 4, i.e. using (4.2) and (3.2), with the parameter $\beta=20$,

(3) denotes the ratio of the value in (2) to the value in (1),

(4) as in (2) but with $\beta=10$,

(5) denotes the ratio of the value in (4) to the value in (1).

The two sets of approximations to ${ }_{0} \delta(u, t)$ shown in Table 2, i.e. (2) and (4), have been calculated using the method for discretizing the individual claim amount distribution given by DE VYLDER and GoovaERTS (1988, Section 8). In the former case it is exactly the same discretization, in the latter case only the parameter $\beta$ is different.

We make the following comments about Table 2 :

(i) The approximations to ${ }_{0} \delta(u, t)$ are always larger than the correct values. This is not surprising since we are using discrete time survival probabilities as approximations to continuous time survival probabilities. This is the problem that DE VYLDER and GoovaERTS (1988) were trying to alleviate by "averaging". See Section 2.

(ii) The relative error in the approximation to ${ }_{0} \delta(u, t)$ for $\beta=10$ is consistently about twice the relative error for $\beta=20$. We would expect the relative error for $\beta=10$ to be larger since it involves a "coarser" discretization of the individual claim amount distribution and also involves "checking for survival" less frequently.

(iii) Where values of $(u, t)$ are given in both Table 1 and Table 2 , the approximations to ${ }_{0} \delta(u, t)$ given by formulae (2.1) and (2.2) (i.e. values (3) in Table 1) can be compared with the approximations given by formulae (4.2) and (3.2) with $\beta=20$, (i.e. values (2) in Table 2). (These values can reasonably be compared since they use precisely the same discretization of the individual claim amount distribution.) It can be seen that the

TABLE 2 (See Section 5 for details)

\begin{tabular}{lllllll}
\hline & & $t=1$ & $t=5$ & $t=10$ & $t=20$ & $t=40$ \\
\hline$u=0$ & $(1)$ & 0.5366 & 0.2804 & 0.2146 & 0.1682 & 0.1362 \\
& $(2)$ & 0.5515 & 0.2921 & 0.2239 & 0.1757 & 0.1423 \\
& $(3)$ & 1.0278 & 1.0417 & 1.0433 & 1.0446 & 1.0455 \\
& $(4)$ & 0.5660 & 0.3036 & 0.2332 & 0.1831 & 0.1485 \\
& $(5)$ & 1.0548 & 1.0827 & 1.0867 & 1.0886 & 1.0903 \\
& & & & & 0.3094 & 0.2529 \\
& $(1)$ & 0.7619 & 0.4881 & 0.3874 & 0.3160 & 0.2584 \\
& $(2)$ & 0.7699 & 0.4971 & 0.3953 & 1.0213 & 1.0217 \\
& $(3)$ & 1.0105 & 1.0184 & 1.0204 & 1.0213 \\
& $(4)$ & 0.7775 & 0.5059 & 0.4030 & 0.3224 & 0.2638 \\
& $(5)$ & 1.0205 & 1.0365 & 1.0403 & 1.0420 & 1.0431
\end{tabular}


TABLE 2 (See Section 5 for details)

\begin{tabular}{|c|c|c|c|c|c|c|}
\hline & & $t=1$ & $t=5$ & $t=10$ & $t=20$ & $t=40$ \\
\hline$u=2$ & $\begin{array}{l}(1) \\
(2) \\
(3) \\
(4) \\
(5)\end{array}$ & $\begin{array}{l}0.8803 \\
0.8844 \\
1.0047 \\
0.8883 \\
1.0091\end{array}$ & $\begin{array}{l}0.6456 \\
0.6522 \\
1.0102 \\
0.6587 \\
1.0203\end{array}$ & $\begin{array}{l}0.5309 \\
0.5373 \\
1.0121 \\
0.5435 \\
1.0237\end{array}$ & $\begin{array}{l}0.4327 \\
0.4383 \\
1.0129 \\
0.4439 \\
1.0259\end{array}$ & $\begin{array}{l}0.3574 \\
0.3623 \\
1.0137 \\
0.3670 \\
1.0269\end{array}$ \\
\hline$u=3$ & $\begin{array}{l}(1) \\
(2) \\
(3) \\
(4) \\
(5)\end{array}$ & $\begin{array}{l}0.9409 \\
0.9429 \\
1.0021 \\
0.9449 \\
1.0043\end{array}$ & $\begin{array}{l}0.7605 \\
0.7652 \\
1.0062 \\
0.7698 \\
1.0122\end{array}$ & $\begin{array}{l}0.6469 \\
0.6520 \\
1.0079 \\
0.6569 \\
1.0155\end{array}$ & $\begin{array}{l}0.5388 \\
0.5436 \\
1.0089 \\
0.5483 \\
1.0176\end{array}$ & $\begin{array}{l}0.4503 \\
0.4546 \\
1.0095 \\
0.4588 \\
1.0189\end{array}$ \\
\hline$u=4$ & $\begin{array}{l}(1) \\
(2) \\
(3) \\
(4) \\
(5)\end{array}$ & $\begin{array}{l}0.9712 \\
0.9722 \\
1.0010 \\
0.9732 \\
1.0021\end{array}$ & $\begin{array}{l}0.8416 \\
0.8449 \\
1.0039 \\
0.8481 \\
1.0077\end{array}$ & $\begin{array}{l}0.7386 \\
0.7425 \\
1.0053 \\
0.7464 \\
1.0106\end{array}$ & $\begin{array}{l}0.6289 \\
0.6329 \\
1.0064 \\
0.6369 \\
1.0127\end{array}$ & $\begin{array}{l}0.5325 \\
0.5363 \\
1.0071 \\
0.5399 \\
1.0139\end{array}$ \\
\hline$u=5$ & $\begin{array}{l}(1) \\
(2) \\
(3) \\
(4) \\
(5)\end{array}$ & $\begin{array}{l}0.9862 \\
0.9867 \\
1.0005 \\
0.9871 \\
1.0009\end{array}$ & $\begin{array}{l}0.8973 \\
0.8996 \\
1.0026 \\
0.9017 \\
1.0049\end{array}$ & $\begin{array}{l}0.8094 \\
0.8125 \\
1.0038 \\
0.8154 \\
1.0074\end{array}$ & $\begin{array}{l}0.7044 \\
0.7078 \\
1.0048 \\
0.7110 \\
1.0094\end{array}$ & $\begin{array}{l}0.6046 \\
0.6079 \\
1.0055 \\
0.6111 \\
1.0108\end{array}$ \\
\hline$u=6$ & $\begin{array}{l}(1) \\
(2) \\
(3) \\
(4) \\
(5)\end{array}$ & $\begin{array}{l}0.9934 \\
0.9937 \\
1.0003 \\
0.9939 \\
1.0005\end{array}$ & $\begin{array}{l}0.9346 \\
0.9361 \\
1.0016 \\
0.9375 \\
1.0031\end{array}$ & $\begin{array}{l}0.8631 \\
0.8654 \\
1.0027 \\
0.8675 \\
1.0051\end{array}$ & $\begin{array}{l}0.7668 \\
0.7696 \\
1.0037 \\
0.7722 \\
1.0070\end{array}$ & $\begin{array}{l}0.6674 \\
0.6703 \\
1.0043 \\
0.6730 \\
1.0084\end{array}$ \\
\hline$u=7$ & $\begin{array}{l}(1) \\
(2) \\
(3) \\
(4) \\
(5)\end{array}$ & $\begin{array}{l}0.9969 \\
0.9970 \\
1.0001 \\
0.9971 \\
1.0002\end{array}$ & $\begin{array}{l}0.9591 \\
0.9600 \\
1.0009 \\
0.9609 \\
1.0019\end{array}$ & $\begin{array}{l}0.9031 \\
0.9047 \\
1.0018 \\
0.9063 \\
1.0035\end{array}$ & $\begin{array}{l}0.8179 \\
0.8201 \\
1.0027 \\
0.8222 \\
1.0053\end{array}$ & $\begin{array}{l}0.7219 \\
0.7243 \\
1.0033 \\
0.7267 \\
1.0066\end{array}$ \\
\hline$u=8$ & $\begin{array}{l}(1) \\
(2) \\
(3) \\
(4) \\
(5)\end{array}$ & $\begin{array}{l}0.9986 \\
0.9986 \\
1.0000 \\
0.9987 \\
1.0001\end{array}$ & $\begin{array}{l}0.9747 \\
0.9753 \\
1.0006 \\
0.9759 \\
1.0012\end{array}$ & $\begin{array}{l}0.9322 \\
0.9334 \\
1.0013 \\
0.9346 \\
1.0026\end{array}$ & $\begin{array}{l}0.8590 \\
0.8608 \\
1.0021 \\
0.8625 \\
1.0041\end{array}$ & $\begin{array}{l}0.7687 \\
0.7708 \\
1.0027 \\
0.7728 \\
1.0053\end{array}$ \\
\hline$u=9$ & $\begin{array}{l}(1) \\
(2) \\
(3) \\
(4) \\
(5)\end{array}$ & $\begin{array}{l}0.9993 \\
0.9994 \\
1.0001 \\
0.9994 \\
1.0001\end{array}$ & $\begin{array}{l}0.9846 \\
0.9850 \\
1.0004 \\
0.9854 \\
1.0008\end{array}$ & $\begin{array}{l}0.9532 \\
0.9541 \\
1.0009 \\
0.9549 \\
1.0018\end{array}$ & $\begin{array}{l}0.8919 \\
0.8933 \\
1.0016 \\
0.8947 \\
1.0031\end{array}$ & $\begin{array}{l}0.8087 \\
0.8105 \\
1.0022 \\
0.8122 \\
1.0043\end{array}$ \\
\hline$u=10$ & $\begin{array}{l}(1) \\
(2) \\
(3) \\
(4) \\
(5)\end{array}$ & $\begin{array}{l}0.9997 \\
0.9997 \\
1.0000 \\
0.9997 \\
1.0000\end{array}$ & $\begin{array}{l}0.9908 \\
0.9910 \\
1.0002 \\
0.9912 \\
1.0004\end{array}$ & $\begin{array}{l}0.9681 \\
0.9687 \\
1.0006 \\
0.9693 \\
1.0012\end{array}$ & $\begin{array}{l}0.9179 \\
0.9190 \\
1.0012 \\
0.9200 \\
1.0023\end{array}$ & $\begin{array}{l}0.8427 \\
0.8442 \\
1.0018 \\
0.8456 \\
1.0034\end{array}$ \\
\hline
\end{tabular}


two sets of values are identical up to the fourth significant figure and hence it appears as though the two algorithms are "as accurate as each other".

\subsection{Some comments on numerical stability}

The algorithms specified by formulae (2.1) and (2.2) and by formulae (4.2) and (3.2) involve a considerable number of numerical operations. In such situations the numerical stability of an algorithm must be of concern. (An algorithm is numerically unstable if small errors in individual numerical operations, as a result of machine rounding for example, can combine to give uncontrollably large errors in the final results. See, for example, ConTE and DE Boor (1980)).

DE Vylder and Goovaerts (1988, Section 5) demonstrate that the algorithm specified by formulae (2.1) and (2.2) is numerically stable. However, the algorithm specified by formulae (4.2) and (3.2) does not appear to be stable. The authors have experienced difficulties (e.g. calculated $\delta$ values outside the range zero to one) when using formulae (4.2) and (3.2) to approximate ${ }_{0} \delta(u, t)$ for values of $u$ greater than about 30 with individual claim amounts having an exponential distribution (with mean 1). These difficulties seem to occur:

(i) independently of the value of $t$, and,

(ii) independently of the value of $\beta$.

This last observation may be a little surprising since reducing the value of $\beta$ reduces the number of numerical operations required to approximate ${ }_{0} \delta(u, t)$ for given values of $u$ and $t$.

We can prove the following result concerning the error in the calculation of $\delta(u, t)$ using formulae (4.2) and (3.2). Instead of $\delta(u, t)$, let us assume that $\hat{\delta}(u, t)$ has been calculated, due to rounding errors, and that $\hat{\delta}(u, t)$ satisfies (3.2). We define $\varepsilon(u, t)$ to be the error in the calculation of $\delta(u, t)$, so that

$$
\varepsilon(u, t)=\delta(u, t)-\hat{\delta}(u, t)
$$

and, for given $u$ and $t, \varepsilon$ to be the modulus of the maximum error in the calculation of $\delta(0, \tau)$ for $\tau=t, t+1, \ldots, t+u$, so that

$$
\varepsilon=\max _{t \leq \tau \leq t+u}|\varepsilon(0, \tau)|
$$

Then we can show that

$$
\begin{array}{ll}
|\varepsilon(w, \tau)| \leq \varepsilon\left(2 g_{0}^{-1}\right)^{w} & \text { for } \quad \tau=t, t+1, \ldots, t+u, \\
& \text { and } \quad w=0,1,2, \ldots, t+u-\tau .
\end{array}
$$


Proof: The proof is by induction, working back from $\tau=t+u$ to $\tau=t$. Note first that (5.1) holds for $\tau=t+u$ since the only possible value for $w$ in this case is 0 and

$$
|\varepsilon(0, t+u)| \leq \varepsilon=\varepsilon\left(2 g_{0}^{-1}\right)^{0}
$$

by definition of $\varepsilon$. Now assume (5.1) holds for $\tau=\tau^{*}+1$, for some $\tau^{*}$, so that

$$
\left|\varepsilon\left(w, \tau^{*}+1\right)\right| \leq \varepsilon\left(2 g_{0}^{-1}\right)^{w} \text { for } w=0,1, \ldots, t+u-\tau^{*}-1
$$

We have to show that

$$
\left|\varepsilon\left(w, \tau^{*}\right)\right| \leq \varepsilon\left(2 g_{0}^{-1}\right)^{w} \text { for } w=0,1, \ldots, t+u-\tau^{*}
$$

to complete the induction. We shall prove (5.3) by induction on $w$. Note that (5.3) holds for $w=0$ by definition of $\varepsilon$. Suppose (5.3) holds for $w \leq w^{*}$ for some $w^{*}$, where $0 \leq w^{*}<t+u-\tau^{*}$. From (3.2) the basic equation satisfied by $\varepsilon\left(w^{*}+1, \tau^{*}\right)$ is

$$
\varepsilon\left(w^{*}+1, \tau^{*}\right)=g_{0}^{-1}\left\{\varepsilon\left(w^{*}, \tau^{*}+1\right)-\sum_{i=1}^{w^{*}+1} g_{i} \varepsilon\left(w^{*}+1-i, \tau^{*}\right)\right\}
$$

from which we have

$$
\begin{aligned}
\left|\varepsilon\left(w^{*}+1, \tau^{*}\right)\right| & \leq g_{0}^{-1}\left\{\left|\varepsilon\left(w^{*}, \tau^{*}+1\right)\right|-\sum_{i=1}^{w^{*}+1} g_{i}\left|\varepsilon\left(w^{*}+1-i, \tau^{*}\right)\right|\right\} \\
& \leq g_{0}^{-1}\left\{\varepsilon\left(2 g_{0}^{-1}\right)^{w^{*}}+\sum_{i=1}^{w^{*}+1} g_{i} \varepsilon\left(2 g_{0}^{-1}\right)^{w^{*}+1-i}\right\} \\
& \leq g_{0}^{-1}\left\{\varepsilon\left(2 g_{0}^{-1}\right)^{w^{*}}+\varepsilon\left(2 g_{0}^{-1}\right)^{w^{*}}\right\} \\
& =\varepsilon\left(2 g_{0}^{-1}\right)^{w^{*}+1}
\end{aligned}
$$

using (5.2) and (5.3). Hence, by induction, (5.3) holds for $w=w^{*}+1$ and hence, also by induction, (5.1) holds for $\tau=\tau^{*}$.

This result is somewhat unsatisfactory since it gives only an upper bound for $|\varepsilon(u, t)|$ rather than more detailed information about how this error behaves, and also because for large values of $u$ it may very well be greater than 1 . Note that for values of $\beta$ used in this paper $g_{0}$ is close to 1 . For example, in Table 2 with $\beta=20$ the value of $g_{0}$ is 0.95663 , but the maximum value of $w$ is 200 so that $\varepsilon$ will need to be very small indeed for the upper bound in (5.1) to be less than 1 !

However, the result does have some interesting features:

(a) The upper bound for $|\varepsilon(u, t)|$ is explicitly a function of $u$, not of $t$ (although $\varepsilon$ itself will be a function of $t$ ). See remark (i) earlier in this section. 
(b) Suppose we wish to approximate ${ }_{0} \delta(u, t)$ for some given $u$ and $t$ using formulae (4.2) and (3.2). Suppose further that we do this twice using different values for $\beta$, one twice the value of the other, say $\beta$ and $\hat{\beta}=2 \beta$. Then, in an obvious notation,

$$
g_{0}=e^{-1 /(1+\theta) \beta} ; \hat{g}_{0}=e^{-1 / 2(1+\theta) \beta}
$$

The value of ${ }_{0} \delta(u, t)$ is approximated by $\delta(u \beta,(1+\theta) \beta \mathrm{t})$ and $\delta(2 u \beta, 2(1+\theta) \beta t)$ in each case and the upper bounds given by (5.1) for the errors will be

$$
\begin{aligned}
|\varepsilon(u \beta,(1+\theta) \beta t)| & \leq \varepsilon\left(2 e^{1 /(1+\theta) \beta}\right)^{u \beta} \\
& =\varepsilon 2^{u \beta} e^{u /(1+\theta)} \\
|\varepsilon(2 u \beta, 2(1+\theta) \beta t)| & \leq \hat{\varepsilon}\left(2 e^{1 / 2(1+\theta) \beta}\right)^{2 u \beta} \\
& =\hat{\varepsilon} 2^{2 u \beta} e^{u /(1+\theta)}
\end{aligned}
$$

so that one component of the upper bound is independent of $\beta$. See remark (ii) earlier in this section.

\subsection{A pragmatic solution to the problem of instability}

We can deal with the problem of numerical instability resulting from the use of formulae (4.2) and (3.2), at least superficially, by constraining the results to behave properly. Consider formula (4.2) first. We know that

$$
0<\delta(0, t+1) \leq \delta(0, t) \leq 1
$$

for any $t \geq 0$. Let $\hat{\delta}(0, t)$ be the value calculated using (4.2). Rather than use this value in formula (3.2) we can use $\delta^{\prime}(0, t)$ where

$$
\delta^{\prime}(0, t)=\min \left\{1, \max \left(0, \min \left(\hat{\delta}(0, t), \delta^{\prime}(0, t-1)\right)\right)\right\} \quad \text { for } \quad t \geq 1
$$

In our numerical examples, we did not experience stability problems in the calculation of $\delta(0, t)$.

We can adjust (3.2) in a similar fashion. For $u \geq 1$ the constraints on $\delta(u, t)$ are

$$
0 \leq \max \{\delta(u, t+1), \delta(u-1, t)\} \leq \delta(u, t) \leq 1
$$

Let $\delta^{\prime}($,) denote the (constrained) value of $\delta($,$) actually used and, for given u$ and $t$, let $\delta(u, t)$ be the "value" of $\delta(u, t)$ calculated using (3.2) with $\delta^{\prime}(u-1, t+1)$ and $\delta^{\prime}(u-1, t)$ appearing on the right hand side. Then

$$
\delta^{\prime}(u, t)=\min \left\{1, \max \left(\hat{\delta}(u, t), \delta^{\prime}(u, t+1), \delta^{\prime}(u-1, t)\right)\right\}
$$

(At this stage the reader could be forgiven for thinking that we are treating the symptoms of instability rather than the disease itself!)

Table 3 shows values of, and approximations to, ${ }_{0} \delta(u, t)$ for larger values of $u$ and $t$ than those in Table 2. The premium loading factor $\theta$ is 0.1 and, as in our previous Tables, individual claim amounts are exponentially distributed. 
TABLE 3 (See Section 5 for details)

\begin{tabular}{lcccc}
\hline \hline & & $t=50$ & $t=100$ & $t=150$ \\
\hline$u=0$ & $(1)$ & 0.1284 & 0.1100 & 0.1028 \\
& $(2)$ & 0.1399 & 0.1200 & 0.1121 \\
& $(3)$ & 1.0896 & 1.0909 & 1.0905 \\
$u=11$ & $(1)$ & 0.8467 & 0.7724 & 0.7361 \\
& $(2)$ & 0.8493 & 0.7753 & 0.7390 \\
& $(3)$ & 1.0031 & 1.0038 & 1.0039 \\
$u=22$ & $(1)$ & 0.9844 & 0.9562 & 0.9352 \\
& $(2)$ & 0.9847 & 0.9568 & 0.9359 \\
& $(3)$ & 1.0003 & 1.0006 & 1.0007 \\
$u=33$ & $(1)$ & 0.9990 & 0.9937 & 0.9870 \\
& $(2)$ & 0.9993 & 0.9940 & 0.9875 \\
& $(3)$ & 1.0003 & 1.0003 & 1.0005 \\
$u=44$ & $(1)$ & 1.0000 & 0.9993 & 0.9979 \\
& $(2)$ & 1.0000 & 1.0000 & 1.0000 \\
& $(3)$ & 1.0000 & 1.0007 & 1.0021 \\
$u=55$ & $(1)$ & 1.0000 & 0.9999 & 0.9997 \\
& $(2)$ & 1.0000 & 1.0000 & 1.0000 \\
& $(3)$ & 1.0000 & 1.0001 & 1.0003 \\
\hline \multirow{3}{*}{$\begin{array}{l}3 \\
*\end{array}$} & & & &
\end{tabular}

The key to Table 3 is as follows:

(1) denotes the exact value of ${ }_{0} \delta(u, t)$ given by SEAL (1978, Table 2.4),

(2) denotes the approximation ${ }_{0} \delta(u, t)$ calculated using formulae (4.2) and (3.2) together with the adjustments given by (5.4) and (5.5),

(3) denotes the ratio (2)/(1).

The values in (2) have been calculated using $\beta=10$ and the same discretization of the individual claim amount distribution as in our previous examples.

We make the following comments about Table 3 :

(i) The relative errors follow the same general pattern as those in Table 2, i.e. increasing with $t$ and decreasing with $u$, although the pattern is somewhat less regular than it was in Table 2.

(ii) The magnitudes of the relative errors are consistent with those for $\beta=10$ in Table 2; in particular, introducing the constraints given by (5.4) and (5.5) has not made our approximations to ${ }_{0} \delta(u, t)$ noticeably less accurate.

\section{A TRUNCATION PROCEDURE}

In their paper DE Vylder and GoovaerTs (1988, Section 5) show how the number of calculations, and hence the amount of computer time, involved in 
the calculation of $\delta(u, t)$ using formulae (2.1) and (2.2) can be reduced in such a way that the resulting error is bounded. This truncation procedure requires the specification of a parameter which, in their numerical example, De Vylder and Goovaerts take to be $\frac{1}{3} \times 10^{-6}$. (We have used this truncation procedure with the same parameter value for the calculation of the values of $\delta(u, t)$ in Table 1.) Even with the help of this truncation procedure, we have found that, typically for very small values of $u$, the algorithm specified by formulae (2.1) and (2.2) can sometimes take more computer time to calculate $\delta(u, t)$ than the algorithm specified by (4.2) and (3.2). Even so, the calculation of $\delta(u, t)$ using (4.2) and (3.2), and in particular the calculation of values of $\delta(0, t)$ using (4.2), can require a considerable amount of computer time. However, it is possible to limit the number of calculations involved in the calculation of $\delta(0, t)$ in such a way that the resulting error is bounded, as we show below.

Recall that $Y_{i}$ denotes the amount of the $i$-th individual claim and that $b_{k}$ denotes $P\left[Y_{i}=k\right]$ for $k=0,1,2, \ldots$ We introduce the following notation:

$$
\begin{gathered}
B(k)=P\left[Y_{i} \leq k\right] \text { for } k=0,1, \ldots \\
B^{* n}(k)=P\left[Y_{1}+Y_{2}+\ldots+Y_{n} \leq k\right] \text { for } k=0,1, \ldots
\end{gathered}
$$

Suppose $\varepsilon, 0<\varepsilon<1$, is given. We define $k_{0}$ to be the smallest integer such that

$$
B\left(k_{0}\right)>1-\varepsilon
$$

The random variables $\left\{Y_{i, \varepsilon}\right\}_{i=1}^{\infty}$ are defined as follows:

$$
\begin{aligned}
& Y_{i, \varepsilon}=Y_{i} \quad \text { if } \quad Y_{i} \leq k_{0} \\
& =\infty \quad \text { if } \quad Y_{i}>k_{0}
\end{aligned}
$$

We define

$$
\begin{aligned}
& b_{\varepsilon}(k)=P\left[Y_{i, \varepsilon}=k\right]=b_{k} \quad \text { for } \quad 0 \leq k \leq k_{0} \\
& =0 \quad \text { for } \quad k_{0}<k<\infty \\
& =\sum_{j=k_{0}+1}^{\infty} b_{j} \quad \text { for } k=\infty \\
& B_{\varepsilon}(k)=P\left[Y_{i, \varepsilon} \leq k\right] \\
& B_{\varepsilon}^{* n}(k)=P\left[Y_{1, \varepsilon}+Y_{2, \varepsilon}+\ldots+Y_{n, \varepsilon} \leq k\right]
\end{aligned}
$$

It is an elementary exercise to show that

$$
\begin{aligned}
B^{* n}(k)-n \varepsilon \leq B_{\varepsilon}^{* n}(k) \leq B^{* n}(k) \quad \text { for } \quad k=0,1,2, \ldots \\
\text { and } \quad n=0,1,2, \ldots
\end{aligned}
$$

Recall that $F(j, t)$ is the probability that the aggregate claims up to time $t$ do not exceed $j$. Define $F_{\varepsilon}(j, t)$ to be the corresponding distribution function with 
individual claim sizes given by $Y_{i, \varepsilon}$ rather than $Y_{i}$, and $\delta_{\varepsilon}(0, t)$ to be the appropriate survival probability for this process. Then

$$
\begin{gathered}
F(j, t)-\lambda t \varepsilon \leq F_{\varepsilon}(j, t) \leq F(j, t) \\
\delta(0, t)-\lambda(t+1) \varepsilon g_{0}^{-1} \leq \delta_{\varepsilon}(0, t) \leq \delta(0, t)
\end{gathered}
$$

for $t=1,2, \ldots$, and $j=0,1,2, \ldots$ Formula (6.2) follows from (6.1) and from noting that

$$
F(j, t)=\sum_{n=0}^{\infty} e^{-\lambda t} \frac{(\lambda t)^{n}}{n !} B^{* n}(j)
$$

with a corresponding formula for $F_{\varepsilon}(j, t)$. Formula (6.3) follows from (6.2) and (4.2).

The calculation of $\delta(0, t)$ and $\delta_{\varepsilon}(0, t)$ require the calculation of $F(j, t+1)$ and $F_{\varepsilon}(j, t+1)$ respectively, for $j=0,1,2, \ldots, t$, and these latter calculations are carried out using PANJER's (1981) recursion formula. There can be a considerable saving of computer time in using $\delta_{\varepsilon}(0, t)$ as an approximation to $\delta(0, t)$ since $F_{\varepsilon}(j, t+1)$ may be based on a risk process with considerably fewer possible values for an individual claim.

\section{CALCULATION OF INFINITE TIME SURVIVAL PROBABILITIES}

\subsection{A recursive formula for the infinite time survival probability}

In this section we shall assume that the mean of an individual claim amount, denoted $m_{1}$, is equal to $\beta$, i.e. that the discretisation of the initial individual claim amount in Section 1 has been done in such a way as to preserve the value of the mean claim amount. This condition is satisfied by the discretisation used in all the numerical examples in this paper. See DE VYLDER and GoovaERTS (1988, Section 7).

The rationale underlying (2.2) can also be applied to infinite time giving

$$
\delta(u-1)=\sum_{i=0}^{u} g_{i} \delta(u-i), \quad \text { where } \quad \delta(u)=\lim _{t \rightarrow \infty} \delta(u, t)
$$

This is simply the infinite time version of (3.1), which can be rearranged to give

$$
\delta(u)=g_{0}^{-1}\left[\delta(u-1)-\sum_{i=1}^{u} g_{i} \delta(u-i)\right]
$$

We could apply formula (3.2) if we could calculate values of $\delta(0, t)$. We can apply (7.2) if we can calculate the value of $\delta(0)$. To do this, we consider the limit as $t \rightarrow \infty$ of formula (4.2), using ideas given in Gerber (1979, p. 113). 
We have that

$$
\begin{aligned}
g_{0} \delta(0, t) & =\frac{1}{t+1} \sum_{j=0}^{t} F(j, t+1) \\
& =\frac{1}{t+1} \sum_{j=0}^{t}[1-(1-F(j, t+1))] \\
& =1-\frac{1}{t+1} \sum_{j=0}^{t}[1-F(j, t+1)] \\
& =1-\frac{1}{t+1} \sum_{j=0}^{\infty}[1-F(j, t+1)]+\frac{1}{t+1} \sum_{j=t+1}^{\infty}[1-F(j, t+1)]
\end{aligned}
$$

The summation in the second term on the right hand side of (7.3) is just the mean of the distribution of $S_{t+1}$. As $S_{t+1}$ has a compound Poisson distribution with Poisson parameter $(t+1) /\left[(1+\theta) m_{1}\right]$, this term reduces to $1 /(1+\theta)$.

Hence,

$$
g_{0} \delta(0, t)=\frac{\theta}{1+\theta}+\frac{1}{t+1} \sum_{j=t+1}^{\infty}[1-F(j, t+1)]
$$

Finally, consider $1-F(j, t+1)=\operatorname{Pr}\left[S_{t+1} \geq j+1\right]$. Now $S_{t+1}$ has mean $(t+1) /(1+\theta)$ and variance $(t+1) m_{2} /\left[(1+\theta) m_{1}\right]$. We can apply Chebychev's inequality as follows:

$$
\begin{aligned}
\operatorname{Pr}\left(S_{t+1} \geq j+1\right) & =\operatorname{Pr}\left[S_{t+1}-\frac{t+1}{1+\theta} \geq j+1-\frac{t+1}{1+\theta}\right] \\
& \leq \operatorname{Pr}\left[\left|S_{t+1}-\frac{t+1}{1+\theta}\right| \geq j+1-\frac{t+1}{1+\theta}\right] \\
& \leq \frac{V\left(S_{t+1}\right)}{\left(j+1-\frac{t+1}{1+\theta}\right)^{2}}
\end{aligned}
$$

Then, $\sum_{j=t+1}^{\infty}[1-F(j, t+1)] \leq V\left(S_{t+1}\right) \sum_{j=t+1}^{\infty}\left(j+1-\frac{t+1}{1+\theta}\right)^{-2}$. 
Consider the sum

$$
\begin{aligned}
S & =\frac{1}{(t+2-\alpha)^{2}}+\frac{1}{(t+3-\alpha)^{2}}+\frac{1}{(t+4-\alpha)^{2}}+\ldots \text { where } \alpha=(t+1) /(1+\theta) \\
& <\frac{1}{(t+2-\alpha)(t+1-\alpha)}+\frac{1}{(t+3-\alpha)(t+2-\alpha)}+\ldots \text { provided that } \theta>0 \\
& =\left(\frac{1}{t+1-\alpha}-\frac{1}{t+2-\alpha}\right)+\left(\frac{1}{t+2-\alpha}-\frac{1}{t+3-\alpha}\right)+\ldots \\
& =\frac{1}{t+1-\alpha}=\frac{1+\theta}{\theta(t+1)}
\end{aligned}
$$

Hence, $\frac{1}{t+1} \sum_{j=t+1}^{\infty}[1-F(j, t+1)] \leq \frac{1}{t+1} V\left(S_{t+1}\right) \frac{1+\theta}{\theta(t+1)}=\frac{1}{t+1} \frac{m_{2}}{\theta m_{1}}$

so that $g_{0} \delta(0, t) \leq \frac{\theta}{1+\theta}+\frac{1}{t+1} \frac{m_{2}}{\theta m_{1}}$.

Finally, as $g_{0} \delta(0, t) \geq \frac{\theta}{1+\theta}$ by (7.4), we see that by letting $t \rightarrow \infty$ we have

$$
\delta(0)=\frac{\theta}{g_{0}(1+\theta)}
$$

Again it is interesting to compare results for our discrete time process with those for the general risk process as specified by (1.1). We note that $\delta^{*}(0)=\theta /(1+\theta)$, which is the same as the ultimate survival probability in continuous time from initial reserve 0 in the general risk process.

Formulae (7.1) and (7.5) correspond to equations (33) and (37) in a paper by DUfRESNE (1988), but he does not consider their numerical application. An earlier reference, also given by Dufresne, is Giezendanner, Straub and Wettenschwiler (1972). An alternative method of finding $\delta(0)$ which does not require equation (4.2) is given in his paper.

We can now apply (7.1) in a recursive manner to calculate survival probabilities starting from

$$
\delta(1)=g_{0}^{-1}\left(1-g_{1}\right) \delta(0)
$$

We can use calculated values of $\delta(\beta u)$ to approximate to

$$
{ }_{0} \delta(u)=\lim _{t \rightarrow \infty}{ }_{0} \delta(u, t)
$$




\subsection{A numerical illustration}

Table 4 shows values of, and approximations to, ${ }_{0} \delta(u)$. As in Tables 2 and 3, individual claims are exponentially distributed and the loading factor, $\theta$, equals 0.1. The discretization of the exponential distribution is as before. The key to Table 4 is as follows:

(1) denotes the exact value of ${ }_{0} \delta(u)$ (see, e.g., SEAL $(1978$, p. 60)),

(2) denotes the approximation to ${ }_{0} \delta(u)$ calculated using formulae (7.2) and (7.5), with $\beta=20$,

(3) denotes the ratio of the value in (2) to the value in (1),

(4) is as (2), but with $\beta=40$,

(5) denotes the ratio of the value in (4) to the value in (1),

(6) is as (2), but with $\beta=100$,

(7) denotes the ratio of the value in (6) to the value in (1).

We make the following comments about Table 4:

(i) The pattern of results is similar to that in Table 2. The approximate values are always greater than the exact values, and as the value of $\beta$ increases, the relative error in the approximation decreases.

(ii) The authors experienced problems in calculating values of $\delta(u, t)$ for values of $u$ greater than about 30 . There were no such problems in calculating values of $\delta(40)$ and $\delta(80)$. However, for larger values of $u$, the same numerical problems as in Section 5.2 exist.

\subsection{Numerical stability}

As in Section 5.3, we can adopt a pragmatic approach and constrain the calculated values of $\delta(u)$ to behave properly. The calculation of $\delta(0)$ does not pose any problems. For $u \geq 1$, we constrain the function $\delta(u)$ to be such that

$$
0 \leq \delta(u-1) \leq \delta(u) \leq 1
$$

TABLE 4 (See Section 7 for details)

\begin{tabular}{rccccccc}
\hline$u$ & $(1)$ & $(2)$ & $(3)$ & $(4)$ & $(5)$ & $(6)$ & $(7)$ \\
\hline 0 & 0.0909 & 0.0950 & 1.0451 & 0.0930 & 1.0231 & 0.0917 & 1.0088 \\
2 & 0.2420 & 0.2454 & 1.0140 & 0.2438 & 1.0074 & 0.2427 & 1.0029 \\
4 & 0.3681 & 0.3709 & 1.0076 & 0.3695 & 1.0038 & 0.3686 & 1.0014 \\
6 & 0.4731 & 0.4754 & 1.0049 & 0.4743 & 1.0025 & 0.4736 & 1.0010 \\
8 & 0.5607 & 0.5626 & 1.0034 & 0.5617 & 1.0018 & 0.5611 & 1.0007 \\
10 & 0.6337 & 0.6353 & 1.0025 & 0.6346 & 1.0014 & 0.6341 & 1.0006 \\
20 & 0.8524 & 0.8531 & 1.0008 & 0.8528 & 1.0005 & 0.8526 & 1.0002 \\
40 & 0.9760 & 0.9761 & 1.0001 & 0.9761 & 1.0001 & 0.9761 & 1.0001 \\
80 & 0.9994 & 0.9994 & 1.0000 & 0.9994 & 1.0000 & 0.9994 & 1.0000 \\
\hline
\end{tabular}


Let $\delta^{\prime}(u)$ denote the constrained value of $\delta(u)$ actually used and, for given $u$, let $\hat{\delta}(u)$ be the "value" of $\delta(u)$ calculated using (7.2) with $\delta^{\prime}$ appearing on the right hand side. Then

$$
\delta^{\prime}(u)=\min \left\{1, \max \left(\delta^{\prime}(u-1), \hat{\delta}(u)\right)\right\}
$$

We can calculate approximate values of ${ }_{0} \delta(u)$ using formulae (7.2) and (7.5), together with the adjustment given by (7.6). We have not produced a table of results because the exact and approximate values (with $\beta=20$ and with $\beta=40$ ) are both 1 to four decimal places for $u \geq 110$.

\section{SOME COMMENTS ON THE DEFINITION OF SURVIVAL}

Our aim in this paper has been to show how to approximate the continuous time probability of survival ${ }_{0} \delta(u, t)$ by the discrete time probability of survival $\delta(u \beta,(1+\theta) \beta t)$. Formulae (4.2) and (3.2) are exact for $\delta(u \beta,(1+\theta) \beta t)$. However, if we regard the latter as an approximation to ${ }_{0} \delta(u, t)$ we find that, being a discrete time approximation to a continuous time probability of survival, it will tend to overstate ${ }_{0} \delta(u, t)$, as noted in comment (i) in Section 5.1 .

If, in addition, the claim amounts have a continuous distribution, as is the case in all the numerical examples considered in this paper, there is a further reason why $\delta(u \beta,(1+\theta) \beta t)$ may overstate the value of ${ }_{0} \delta(u, t)$. This is that for survival to occur according to the former, the surplus need only stay above the value -1 (but could be zero at any time), whereas for survival to occur according to the latter, the surplus must never go below zero, no matter by how little.

For the risk process characterized by (1.2) we defined in Section 4 the survival probability $\delta^{*}(u, t)$ for $u \geq 0$ and $1 \leq t \leq \infty$ as follows:

$$
\delta^{*}(u, t)=\operatorname{Pr}\left[u+\tau-\sum_{i=1}^{N_{\tau}} Y_{i} \geq 1 \quad \text { for } \quad \tau=1,2, \ldots, t\right]
$$

This differs from $\delta(u, t)$ in that for survival it requires the surplus to be strictly greater than zero after time zero. For finite $t, \delta^{*}(0, t)$ can be calculated from formula (4.3). For $t$ equal to infinity, $\delta^{*}(0)$ is equal to $\theta /(1+\theta)$, as explained in Section 7 . For $u$ greater than zero it is clear that:

$$
\delta^{*}(u, t)=\delta(u-1, t)
$$

It could be argued, for the reason given in the second paragraph in this section, that $\delta^{*}(u \beta,(1+\theta) \beta t)$ is a more logical approximation than $\delta(u \beta,(1+\theta) \beta t)$ to ${ }_{0} \delta(u, t)$, although, depending to some extent on the discretization of the claim amount distribution, it may tend to understate ${ }_{0} \delta(u, t)$.

Table 5 shows the results of approximating ${ }_{0} \delta(u, t)$ by $\delta^{*}(u \beta,(1+\theta) \beta t)$ for the risk process with exponentially distributed individual claims, premium loading factor equal to 0.1 and parameter $\beta$ equal to 20 . The key to Table 5 is as follows: 
TABLE 5 (See Section 8 for details)

\begin{tabular}{llllll}
\hline \hline & & $t=10$ & $t=20$ & $t=40$ & $t=\infty$ \\
\hline$u=0$ & $(1)$ & 0.2146 & 0.1682 & 0.1362 & 0.0909 \\
& $(2)$ & 0.2146 & 0.1682 & 0.1362 & 0.0909 \\
& $(3)$ & 1.0000 & 1.0000 & 1.0000 & 1.0000 \\
$u=5$ & $(1)$ & 0.8094 & 0.7044 & 0.6046 & 0.4230 \\
& $(2)$ & 0.8094 & 0.7043 & 0.6045 & 0.4229 \\
& $(3)$ & 1.0000 & 0.9999 & 0.9998 & 0.9998 \\
$u=10$ & $(1)$ & 0.9681 & 0.9179 & 0.8427 & 0.6337 \\
& $(2)$ & 0.9681 & 0.9178 & 0.8426 & 0.6337 \\
& $(3)$ & 1.0000 & 0.9999 & 0.9999 & 1.0000 \\
\hline
\end{tabular}

(1) denotes the exact value of ${ }_{0} \delta(u, t)$ as given by SEAL (1978);

(2) denotes the value of $\delta^{*}(u \beta,(1+\theta) \beta t)$ calculated using the methods of Section $4(u=0$ and $t<\infty)$, of Sections 3 and $4(u>0$ and $t<\infty)$ or of Section $7(t=\infty)$ as appropriate;

(3) denotes the ratio (2)/(1).

The approximations to ${ }_{0} \delta(u, t)$ in Table 5 can be compared with the approximations (for $\beta=20$ ) in Tables 2 and 4 . It can be seen that the approximations in Table 5 are very much better than those in Tables 2 and 4 . One explanation for this may be that two "errors" in the approximation of ${ }_{0} \delta(u, t)$ by $\delta^{*}(u \beta,(1+\theta) \beta t)$, i.e.

(a) understating ${ }_{0} \delta(u, t)$ by redefining survival/ruin, and

(b) overstating ${ }_{0} \delta(u, t)$ by using a discrete time approximation to a continuous time survival probablity,

are working in opposite directions and cancelling each other out.

\section{REFERENCES}

CONTE, S. D. and De Boor, C. (1980) Elementary Numerical Analysis. McGraw Hill-Kogakusha, London.

De Vylder, F. and GoovaerTs, M. J. (1988) Recursive calculation of finite-time ruin probabilities. Insurance: Mathematics and Economics 7, 1-8.

DUfRESNE, F. (1988) Distributions stationnaires d'un système bonus-malus et probabilité de ruine. ASTIN Bulletin 18, 31-46.

Gerber, H.U. (1979) An Introduction to Mathematical Risk Theory. S. S. Huebner Foundation Monograph Series No. 8. Distributed by R. Irwin, Homewood, IL.

Giezendanner, E., Straub, E. and Wettenschwiler, K. (1972) Zur Berechnung von Ruinwahrscheinlichkeiten. Transactions of the 19th International Congress of Actuaries, Oslo, 4, 645-651.

PANJER, H. H. (1981) Recursive evaluation of a family of compound distributions. ASTIN Bulletin 12, 22-26.

Seal, H. L. (1978) Survival Probablities. John Wiley and Sons, New York.

WikstAd, N. (1971) Exemplification of ruin probabilities. ASTIN Bulletin 6, 147-152.

David C.M. Dickson and Howard R. Waters

Department of Actuarial Mathematics and Statistics, Heriot-Watt University, Edinburgh EHI4 4 AS, United Kingdom. 